\title{
A novel approach to simulate the stiffness behaviour of spot welded vehicle structures under multi axial variable amplitude loading
}

\author{
Peter Rösch ${ }^{1, *}$, Halvar Schmidt ${ }^{1}$, and Thomas Bruder $^{1}$ \\ ${ }^{1}$ BMW AG, Knorrstr. 147, 80807 Munich, Germany
}

\begin{abstract}
Under cyclic loading in combination with high local stress levels a change in stiffness of connection points, e.g. spot welds, self-piercing rivets or flow drill screws can be observed. This paper introduces a numerical approach for the simulation of stiffness changes in vehicle structures under multiaxial cyclic loading as a result of the stiffness degradation of individual spot welds. The basic approach is based on a conventional damage accumulation combined with a power law representing the change in the spot weld's stiffness. Instead of an accumulated damage value D a residual stiffness is set as failure criterion. Extensions to the approach are presented for its application to multiaxial loading with variable amplitudes. A conventional multiaxial fatigue test of a complete vehicle, focusing on its rear end including global stiffness and local strain measurements at the beginning, median and at the end of the test are presented and compared to the simulated results. This computation approach allows a more reliable fatigue assessment in comparison to a fatigue analysis which does not consider stiffness changes. The damage propagation after the crack initiation at an individual spot weld is represented more accurately as subsequent changes in the load paths are considered.
\end{abstract}

\section{Introduction}

Strength and stiffness requirements highly influence the dimensions and therefore also the weight of components and structures. In order to meet increasing customer demands and to reduce the overall vehicle emissions, light weight construction is an essential aspect in the vehicle development process. To achieve a lightweight construction a diversity of materials and joining techniques, are applied. Resistance spot welding is one of the most commonly used joining techniques in conventional vehicles. Current research projects, e.g. ALIVE (Advanced High Volume Affordable Lightweighting for Future Electric Vehicles) show a high potential for resistance spot welding or similar techniques (e.g. resistance element welding) to be applied in future vehicle projects [1]. To illustrate the usage of resistance spot welding in the automotive industry, Figure 1 shows the spot welds in a current generation of a sport utility vehicle. In total more than 6500 spot welds are used in the vehicle body in white.

Especially theses joints are known to change their stiffness during fatigue life which can have an impact on the local stress state. For a consequent light weight construction, novel methodologies are needed to predict the material's fatigue behaviour as stiffness changes are not taken into account in currently used fatigue analysis tools yet. Therefore increased safety factors are used in the design process which lead to an unnecessary increase in the overall vehicle weight. As a result of local stiffness changes, fatigue life may be affected positively or negatively. An elongation of fatigue life can occur if, due to changes in the local stiffness, stresses redistribute in areas which have observed lower stresses in the previous fatigue life. Under different conditions a reduction of fatigue life may also be a consequence of degradation. The stiffness behaviour often depends on loading direction and load spectrum, material combination or the used joining technique, respectively. In this paper the basic approach DegraRP [2], which showed promising results for the stiffness degradation simulation under single channel torsional loading, is extended and applied to a complete vehicle simulation in order to take into account multiaxial loading.

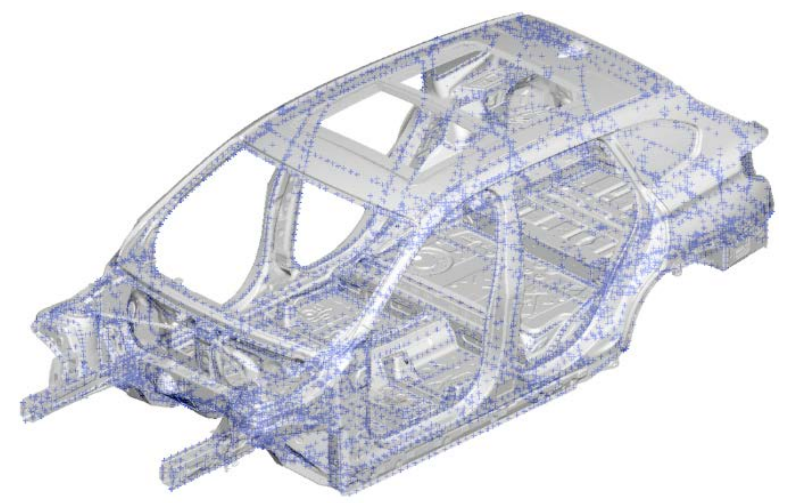

Fig. 1 Spot weld connections in a current sport utility vehicle 


\section{Theoretical Background}

Resistance spot welding is widely used in the automotive industry. The joint between two components is produced by heat, generated through the components resistance against an applied current, and pressure [3]. In the following section the theory of different fatigue assessment approaches of spot welded structures and the methodology DegraRP are introduced briefly.

\subsection{Fatigue assessment of spot welded structures}

The complexity in the assessment of spot welded structures results from various challenges compared to non-welded structures, for example the [4]:

- determination of different material properties and hardnesses within the spot weld (base material, nugget or heat affected zone),

- uncertain residual stresses in the spot weld due to the manufacturing process,

- uncertain micro-geometry on the spot weld's notch,

- unknown crack initiation and propagation areas.

Especially the last bullet point is highly dependent on the local stress state and the load level of the tested specimens and structures and has been focus of a diversity of researchers since the 1970s [5-7].

For the numerical fatigue life assessment of spot welded components the FESPOW concept is often used in the automotive industry $[8,9]$. The spot weld nugget with a diameter $d$ connecting two sheets with the according sheet thickness $t_{1,2}$ is represented by beam elements. Six general forces are determined acting on the nugget. Beam cross-section forces $F_{x i}, F_{y i}, F_{z i}$ and moments $M_{x i}, M_{y i}$ are used to derive the equivalent stress state $\sigma_{v}$ in the base material which will be evaluated during fatigue life, see equations (1)-(7). Torsion of the spot weld itself $M_{z i}$ is not considered in this model.

$$
\begin{gathered}
\boldsymbol{\sigma}_{v 1}=\left(\sigma_{\max , F x 1}+\sigma_{\max , M y 1}\right) \cos (\varphi)+ \\
\left(\sigma_{\max , F y 1}-\sigma_{\max , M x 1}\right) \sin (\varphi)-\sigma_{F z 1} \\
\boldsymbol{\sigma}_{v 2}=\left(\sigma_{\max , F x 2}-\sigma_{\max , M y 2}\right) \cos (\varphi)+ \\
\left(\sigma_{\max , F y 2}+\sigma_{\max , M x 2}\right) \sin (\varphi)+\sigma_{F z 2}
\end{gathered}
$$

The representative equivalent stress $\sigma_{v}$ is the maximum of stress $\sigma_{v 1}$ and $\sigma_{v 2}$ resulting in each sheet. The equivalent stress state is composed of a shear-tension $\left(\sigma_{\max , F x i}, \sigma_{\max , F y i}\right)$, cross-tension $\left(\sigma_{F z i}\right)$ and peel-tension $\left(\sigma_{\max , M x i}, \sigma_{\max , M y i}\right)$ stress portion.

With the FESPOW approach only a single SN curve, derived from a variety of cyclic tests on single spot weld specimens, is necessary compared to an approach in which the stress in the neighboring elements of the nugget is evaluated (FEMFAT Spot) [10]. The associated Finite Element modelling for both approaches is presented in Figure 2. Using the FEMFAT Spot approach different SN curves are applied for damage accumulation, depending on the local stress tensor (tension/compression, shear or a combination of both). The SN curves are derived using a variety of specimens, e.g. H or KS specimens. In addition to the introduced

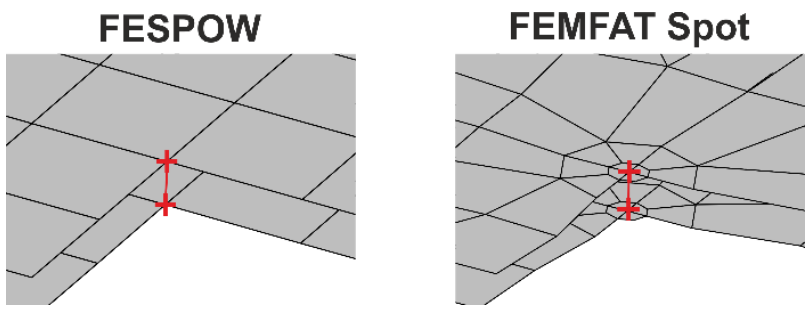

Fig. 2 FESPOW vs. FEMFAT Spot concept

assessment approaches for spot welds notch-stress or strain approaches are available but rarely used in the automotive industry.

\subsection{Degradation simulation of uniaxial loaded structures}

To simulate stiffness degradation during fatigue life an incremental computation process - DegraRP - is used. Figure 3 introduces the basic idea of the methodology. A conventional fatigue life simulation (represented with the black outline in Figure 3) is based on the quasi-static FE results, SN curves and damage accumulation using e.g. Miners law and allowable damage sum $D$. Changes in the local stiffness are not considered at all. To be able to consider the stiffness behavior during fatigue life simulation, the stiffness of the FE model needs to be adapted after certain thresholds have been reached (represented through the additional components with a green, dotted outline in Figure 3). A partial damage accumulation computation is conducted after which an evaluation of the current stiffness degradation state is performed. This evaluation is based on a stiffness degradation curve, describing stiffness as a function of the accumulated damage $D$ derived from experimental data. Two additional parameter sets are necessary to determine boundaries for a recomputation with an adapted FE model. The parameter sets can be divided into material and computation (status) parameters. In a conventional fatigue life computation the result is an accumulated damage $D$. During the stiffness degradation simulation the main failure mechanism is described using a residual stiffness of each spot weld [2].

In a first step the stiffness degradation curve is assumed to be a simple power law. The material parameters are determined using a method of least squares to fit the functions parameters to the 


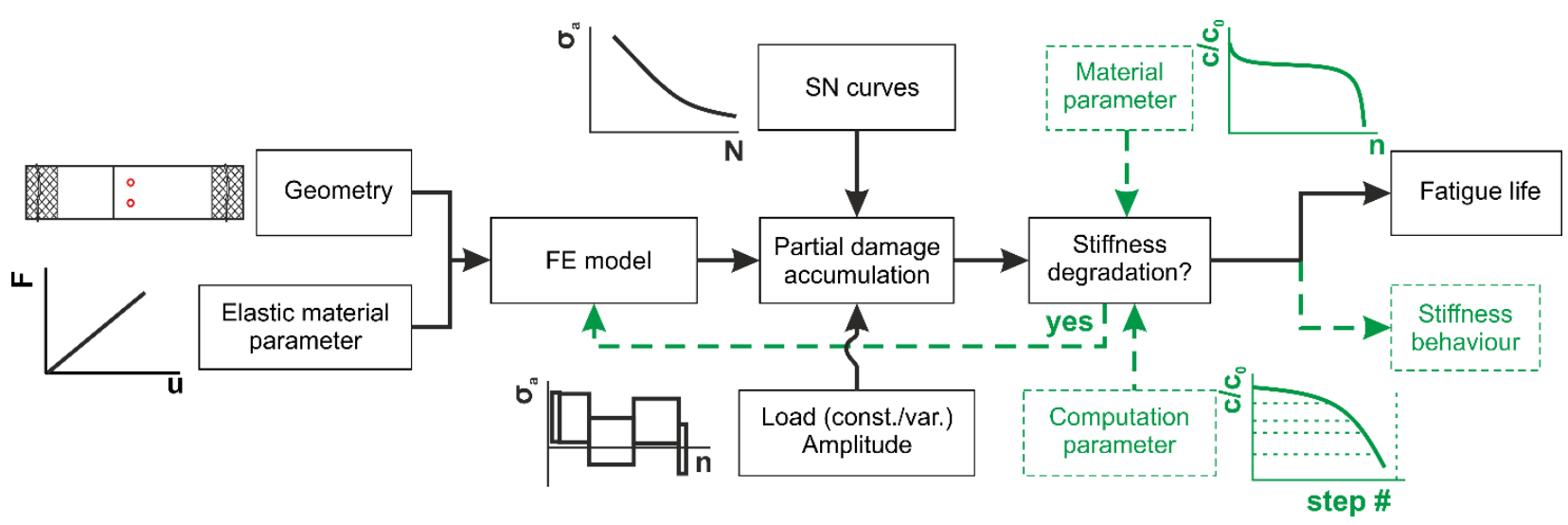

Fig. 3 Numerical stiffness degradation approach DegraRP [2]

experimental data. In a first step the main goal is to show that the principal methodology is capable to simulate stiffness degradation. Deriving a more accurate degradation law will be focus of future research.

The stiffness degradation law is only valid for the loading direction, material combination and weld parameters it has been derived from. The degradation law of another loading direction can be approximated using a combination of known degradation laws. For example for a $45^{\circ}$ direction the degradation law may be approximated using $50 \%$ of a $0^{\circ}$ and $50 \%$ of a $90^{\circ}$ loading direction degradation law. This results in only little testing effort necessary, if applicable. The implemented method uses in total five status parameters for the control of the computation, e.g. when an adaption of the finite element model is necessary or the computation is finished. They have been introduced in detail in previous work not only for joining techniques but also carbon fiber reinforced plastic [2, 11-12].

\section{Experimental test setup and results}

In order to assess the prediction capabilities of the DegraRP methodology a customized experiment is conducted. The main goal of the experiment is to generate failure of certain, highly loaded spot welds during the test, using variable amplitude loads in different load directions. The failure of a spot weld will induce changes in the connection stiffness which effects the stress state in the base material surrounding the spot weld. In the experiment a sport utility vehicles tow bar is loaded in three independent directions $\left(F_{x}, F_{y}\right.$ and $\left.F_{z}\right)$. In the front, the car is mounted in the strut tower. In the back, the rear axle is assembled to the body in white and the fixation is attached to the wheel bearing. An additional support in horizontal direction is attached to one of the front door hinges. A variable amplitude loading is applied which was derived by increasing the loads given by the car loading standard for trailer coupling devices (CARLOS TC) [13]. In order to induce an early failure of certain spot welds the load applied is multiple times higher than it would be under normal testing conditions.

Additionally strain gauges are applied to the vehicles rear end. The strain gauges are used for verification of the complete vehicle's finite element model and the assessment of the local stress state during fatigue testing. The test setup, load directions and the position and orientation of the representative strain gauge rosette \#4 are displayed in Figure 4.

A visual inspection of the rear end structure's damage is conducted after $n / n_{\text {ref }}=25 \%, 50 \%, 75 \%$ and $100 \%$. The strain gauge measurement is conducted prior to the fatigue test, at $n / n_{\text {ref }}=50 \%$ and $n / n_{\text {ref }}=$ $100 \%$. The measurement is performed in each load direction independently. The results measured by the strain gauge 4-3 for each load direction are displayed in Figure 5 and compared to the results of a linear Nastran $^{\mathrm{TM}}$ simulation of the complete vehicle. Each plot is normalized in $\mathrm{x}$ - and $\mathrm{y}$-direction to a representative value (e.g. maximum stress). The experimental data shows an almost linear behavior of the stress state in the base material at strain gauge position \#4. The overall fit of the strains in direction 3 between simulation and testing is acceptable. A similar behavior can be observed in direction 1 and 2. Therefore the representation of the stress state at strain gauge \#4 by the finite element model can be considered accurate. The experimental results show a high alteration of the $\sigma-F$ curves slope through different stages of fatigue life indicating a stiffness degradation. Under the application of a load in $\mathrm{x}$ direction the slope $s_{\sigma}=\Delta \sigma / \Delta F$ increases by $63 \%$ from $s_{\sigma}=0.47$ at the beginning of the experiment to $s_{\sigma}=0.77$ at the end of the test duration $n / n_{\text {ref }}=100 \%$. At the test duration only a minor increase of the slope (by approximately $10 \%$ ) can be observed.

The marked hot spot positions (HS1-HS3) in Figure 4 indicate spot welds which fail during the fatigue testing with disproportionally high loads. A first crack at the

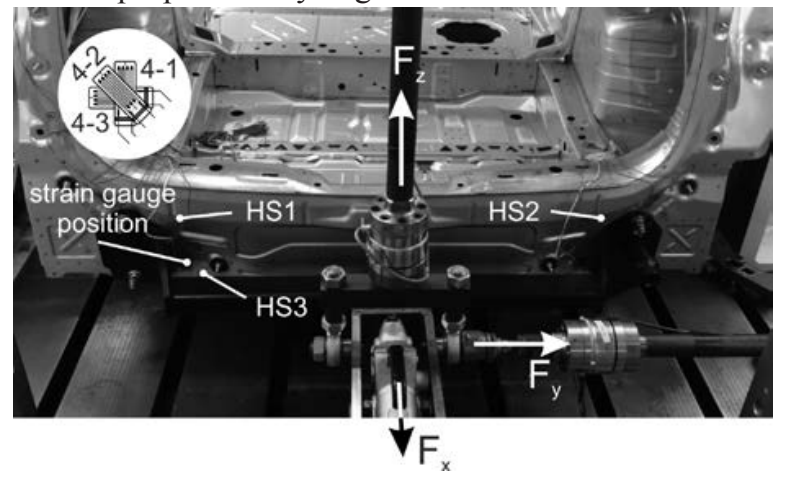

Fig. 4. Test setup, load direction, hot spots, position and orientation of strain gauge $\# 4$ 

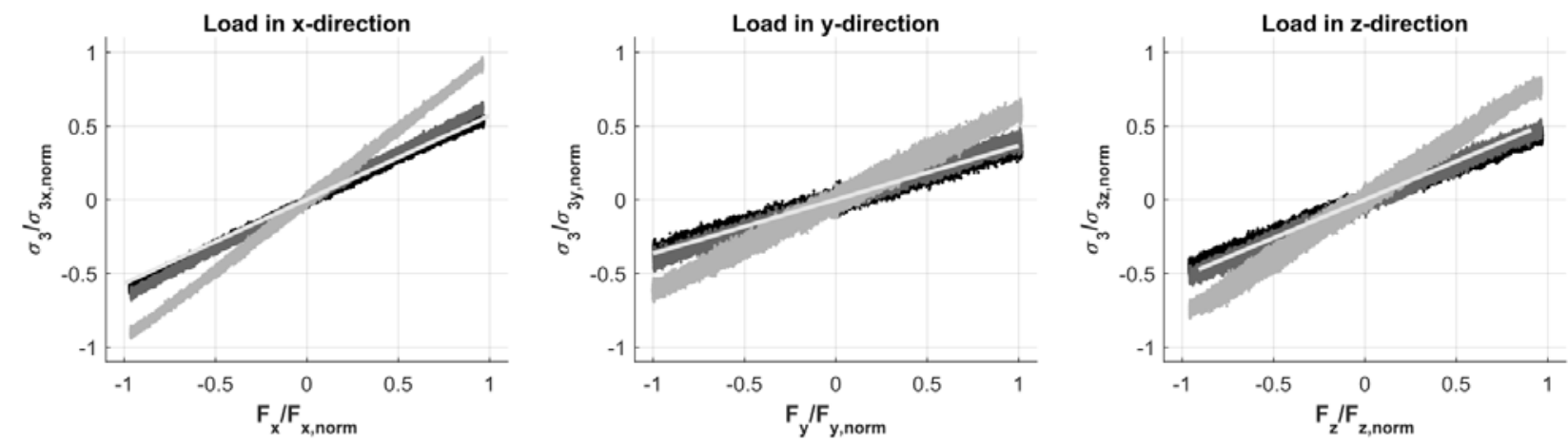

- $\mathrm{n} / \mathrm{n}_{\text {ref }}=0 \bigcirc \mathrm{n} / \mathrm{n}_{\text {ref }}=0.5 \bigcirc \mathrm{n} / \mathrm{n}_{\text {ref }}=1 —$ Nastran linear @ $\mathrm{n} / \mathrm{n}_{\text {ref }}=0$

Fig. 5 Strain gauge 4-3 measurement during different stages of fatigue life and comparison to the initial linear Nastran ${ }^{\mathrm{TM}}$ model

spot weld at HS1 can be observed at approximately $25 \%$ of the testing period.

\section{Simulation of stiffness degradation under multiaxial loading}

In order to simulate stiffness changes under multiaxial, variable amplitude loading several extensions to the existing methodology are necessary which are then tested using a complete vehicle simulation.

\subsection{Extension to the methodology}

The necessary extension of the methodology mainly refers to the evaluation of the stress state at each spot weld in each computations step and the consideration of the effects of multiaxiality on the degradation behavior.

In the existing DegraRP approach one assumption is the quasi-invariability of the stress state at each spot weld under uniaxial loading through the different degradation stages. The portions of stresses due to a certain local loading type (later referred to as stress type with the three main components shear-, cross- and peel tension) on the stiffness degradation are therefore constant through the computation. The associated portions $k_{1}-k_{3}$, see equations (8)-(10), are determined using the equivalent stress established with the FESPOW approach.

$$
\begin{array}{ll}
\text { Shear: } & k_{1}=\frac{\sigma_{v}-\sigma_{v}\left(F_{x i}, F_{y i}=0\right)}{\sigma_{v}} \\
\text { Cross: } & k_{2}=\frac{\sigma_{v}-\sigma_{v}\left(F_{z i}=0\right)}{\sigma_{v}} \\
\text { Peel: } & k_{3}=\frac{\sigma_{v}-\sigma_{v}\left(M_{x i}, M_{y i}=0\right)}{\sigma_{v}}
\end{array}
$$

\section{Example: hat profile (proportional loading)}

A first verification of the assumption is performed looking at the cross section forces after each computation step, simulating the stiffness changes of a hat profile under single channel loading. Looking at the shear-tension parameter $k_{3}$ the average deviation from its initial value (at $D=0$ ) is below $0.1 \%$ for each

computation step. The cross-tension and peel-tension portion of the stress type deviate on average by $1.2 \%$ and $0.05 \%$ in each computation step. Considering the small sensitivity of the methodology to changes in the stress-state parameter $\left(k_{1}-k_{3}\right)$ quasi-invariability of the stress-state through the computation can be assumed for the analyzed example.

\section{Example: tow bar (non-proportional loading)}

Under multi-axial loading the stress state at each spot weld can change in each computation step. To illustrate this behavior, Figure 6 shows the portions of each single stress type (shear-, cross- and peel-tension) at three selected hot spots (HS) and for each loading direction acting on the tow bar individually. Looking at HS1 cross-tension is only relevant under loading in $\mathrm{x}$ -

HS1 HS2

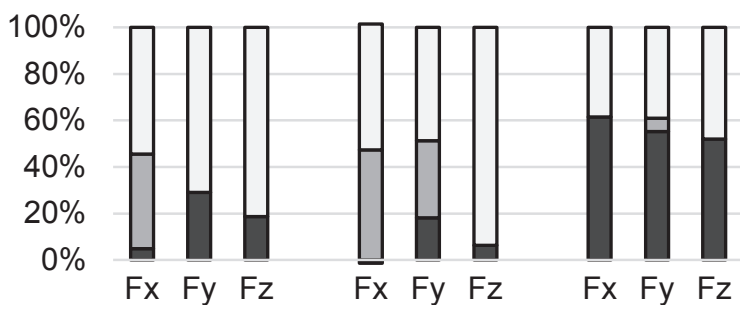

ashear-tension across-tension apeel-tension

Fig. 6 Stress types at selected hot spots under loads acting in directions $(\mathrm{x}, \mathrm{y}, \mathrm{z})$

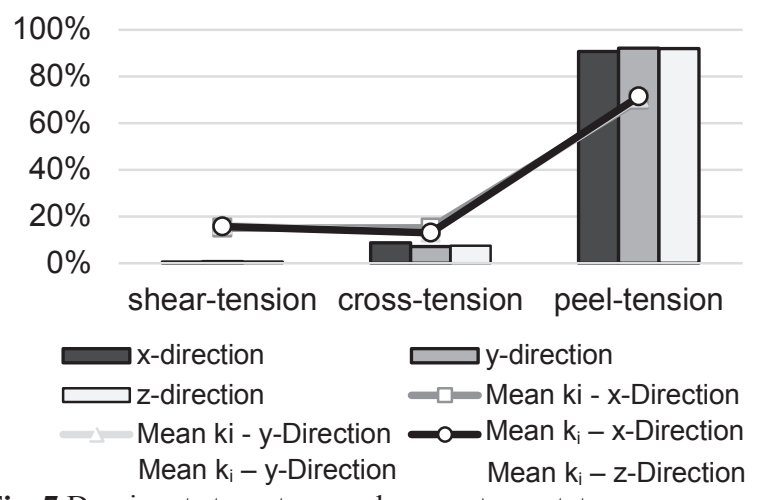

Fig. 7 Dominant stress type and mean stress state on average of all spot welds under loads acting in different directions 


$$
k_{i}=\sum_{t_{j}} k_{i}\left(t_{j}\right) \cdot \frac{\Delta D\left(t_{j}\right)}{\sum_{t_{j}} \Delta D\left(t_{j}\right)} \quad \text { for } \mathrm{i}=1,2,3
$$

direction. At HS2 the stress state is highly dependent of the cross-tension portion and in HS3 the three portions are almost constant. This diversity of changes in the stress state at each spot weld motivates a stress-state evaluation after each computation step. In some cases it can be observed, that the shear-tension leads to a decrease of the equivalent stress, e.g. in HS2 under load in $\mathrm{x}$-direction. As a result the parameter $k_{1}$ is negative and the sum of all parameters $\sum_{i=1}^{3} k_{i}>1$. The implications to the computation of a negative $k_{1}$ could lead to false conclusions as the stiffness could increase in one computation step, depending on the boundary conditions. In this case the parameter $k_{1}$ needs to be set to $k_{1}=0$ and the corresponding $k_{3}$ decreased by the previous $k_{1}$ in order to keep the sum of all parameters at $\sum_{i=1}^{3} k_{i}=1$.

To get an overall impression of the dominating stress type in the complete finite element model, Figure 7 shows the dominating stress type at each spot weld relative to the number of all spot welds under a single constant load at the tow bar $F_{x}, F_{y}$ and $F_{z}$. The dominating stress type is defined by the maximum of $k_{1}$ $k_{3}$ at each spot weld (e.g. in HS1 under a load acting in $\mathrm{x}$-direction, peel tension would be the domination stress type). Over $90 \%$ of the spot-welds in the model are dominated by local peel-tension, followed by cross- and shear-tension. This behavior is almost independent of the loading direction actin at the tow bar. On average over all spot welds the stress state is composed of sheartension $\overline{k_{1}} \approx 15 \%$, cross-tension $\overline{k_{2}} \approx 12 \%$ and peeltension $\frac{1}{k_{3}} \approx 73 \%$. This behavior is also almost independent of the load direction acting at the tow bar.

The second major extension to the methodology takes into account the combination of multiple load directions. In the tow bar test, three unit load cases are applied on the vehicle's tow-bar and superimposed with the CARLOS load-time series and a constant force representing the tow bar's nose weight. Under multiaxial variable amplitude loading the stress state parameters change from load cycle to load cycle not only in their proportions, but also in their relevance as there are load cycles which add almost no damage and load cycles adding a relative high amount of damage to the individual spot weld. Figure 8 shows the necessary computation results at one specific spot weld node during a short time step $(\Delta t=0.5 \mathrm{sec})$ of the conventional fatigue life computation, applying CARLOS Module 3. The radial stress state in the critical plane $\sigma_{\text {rad }}$ gives an overview of the minimum and maximum stresses used for the conventional fatigue life computation. At the four points in time $t_{j}$ (indicated through the dotted line) the damage increment $\Delta D$ is accumulated. As mainly the stress state at the minimum and maximum of the reversal are relevant, the existence of a damage increment $\Delta D$ can be used as a trigger and the height of the increment as a weighting function for

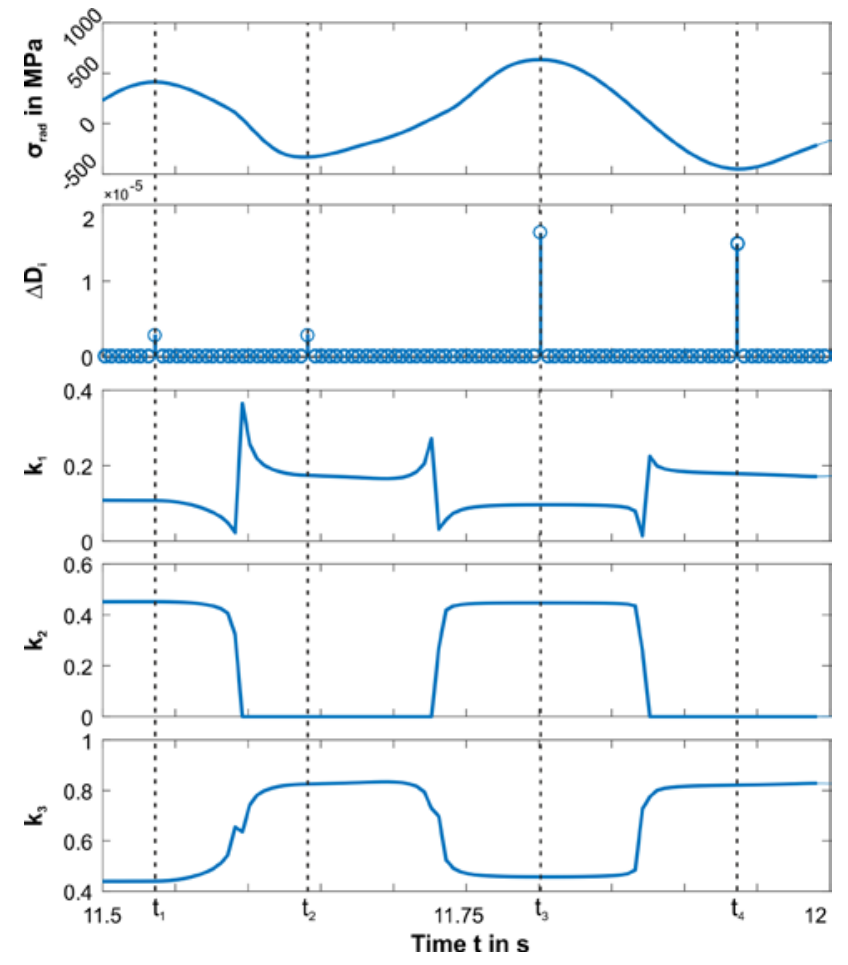

Fig. 8 Clipping of the equivalent stress state, damage increment and stress state parameter $k_{i}$ in CARLOS Module 3

the computation of the overall modules stress state at one specific spot weld, see equation (11).

The computation needs to be performed for the CARLOS Modules 1-3 and for the critical plane of each spot weld.

\subsection{Computation results}

In total four equidistant computation steps $\left(\Delta n / n_{\text {ref }}=25 \%\right)$ were performed applying the extended DegraRP methodology. Prior to the computation a selection of the relevant spot weld connections is conducted in order to minimize the computation effort of the DegraRP methodology. Therefore a conventional fatigue life computation is performed and the accumulated damage is assessed at each spot weld individually with its accumulated damage $D_{s p}$. The threshold of relevance of a single spot weld is set relative to the maximum observable damage in the finite element model $D_{\max }$ to $D_{s p} / D_{\max } \geq 1 e^{-5}$. In total $15 \%$ of the spot welds (considering the test setup and loading conditions at hand) in the vehicle's finite element model are left relevant and are evaluated using the introduced methodology.

Figure 9 shows one of the results of the stiffness degradation computation - the accumulated damage for each computation step. A detail of the vehicles tow bar is illustrated and some of the relevant spot welds (SP1SP4) are highlighted. A redistribution of the accumulated damage after each computation step can be observed. Focusing on SP2 (the spot weld with the highest damage in computation step 1) the damage at the spot weld and the surrounding base material decreases with each 


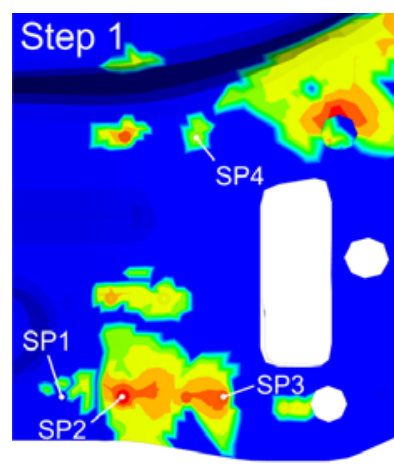

$0-25 \%$

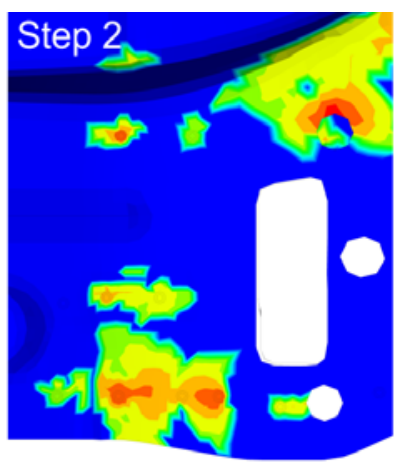

$25-50 \%$

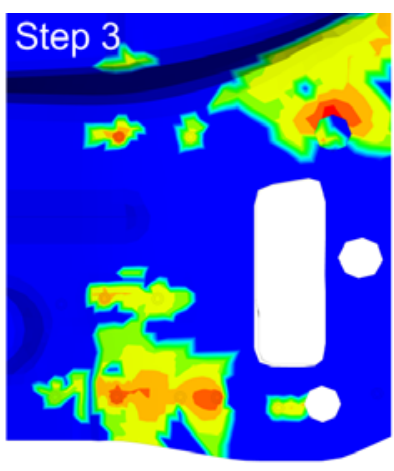

$50-75 \%$

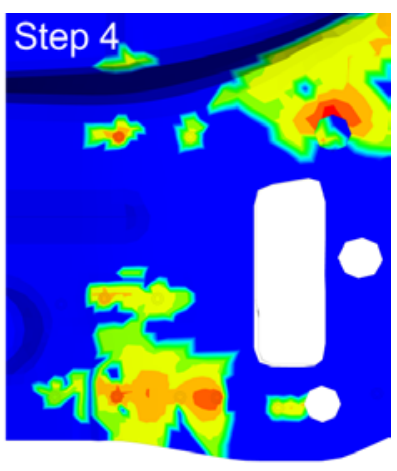

$75-100 \%$

Fig. 9 Fatigue life computation and damage increment in each computation step

computation step due to the simulated stiffness degradation. As a result damage in SP1 and SP3 increases during each computation step. A similar behavior can be observed for SP4. The results show the feasibility and basic simulation capabilities of the extended DegraRP approach.

In order to quantify the simulations result, the stress state at $n / n_{\text {ref }}=50 \%$ and $100 \%$ is compared at the strain gauge \#4. The degradation of the measured strain is compared to the degradation of the simulated strain at the position of strain gauge 4, see Figure 10.

The assessment of the experimental degradation is conducted based on the increase of the introduced slope $s_{\sigma}$. In order to evaluate the simulated degradation at strain gauge \#4, a virtual strain gauge (represented by bar elements with a diameter of $d_{\text {bar }}=0.1 \mathrm{~mm}$ and young modulus $\left.E_{b a r}=210000 \mathrm{MPA}\right)$ is built in the finite element model. At $n / n_{\text {ref }}=50 \%$ the change in the stress-state at strain gauge $\# 4$, induced by changes in the spot weld stiffness, are represented quite accurately for a load in $\mathrm{x}$ - or $\mathrm{z}$-direction and each measuring direction. In y-direction the experimentally observed degradation differs, especially in measuring direction 4-1 and 4-2. A possible explanation for the deviation is the absolute strain at strain gauge \#4 under load in ydirection, which is very small compared to other load and measuring directions. Therefore small changes in the strain results in disproportionally high relative changes.
At $n / n_{\text {ref }}=100 \%$ the computation highly underestimates the degradation at almost every load- and measuring direction, especially in measuring direction 43. The main reason for the deviation is the increasing effect of non-linearity with increasing damage, e.g. evolving cracks in the base material. The consideration of cracks or changes in stiffness of the base material is not possible in the current methodology. To illustrate the limitations of the extended approach a quasi-static computation of the finite element model without any spot welds in the surrounding area of strain gauge \#4 is conducted. The results can be interpreted as a computation with $100 \%$ degraded spot welds near the strain gauge. The resulting degradation of the strain is indicated through the boundaries in the numerical section at $n / n_{\text {ref }}=100 \%$ for selected load- and measuring directions. It is obvious, that even with a complete degradation of the spot welds, the occurring stresses at $n / n_{\text {ref }}=100 \%$ at the location of srain gauge 4-3 cannot be described. For a load applied in x-direction and the measuring direction 4-3 a maximum strain reduction of approximately $42 \%$ can be simulated with the introduced methodology. As a results the measured reduction of over $70 \%$ at strain gauge $\# 4$ cannot be reproduced in a simulation which only considers degradation of the spot weld by the reduction of its beam diameter.
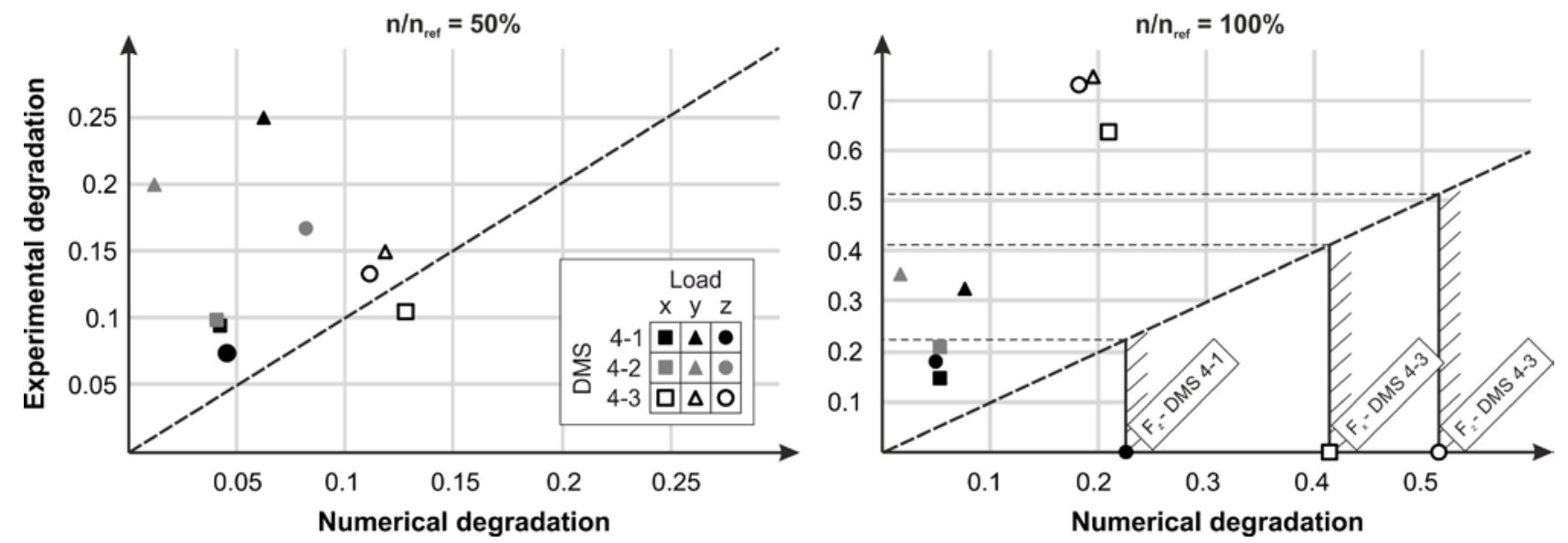

Fig. 10 Comparison of experimental vs. numerical change in strain data - assessed at strain gauge \#4 


\section{Summary and Outlook}

A conventional fatigue life analysis does not consider changes in stiffness of connection points due to fatigue loading. An experimental test conducted on a sport utility vehicles tow bar loaded with a CARLOS TC timeseries shows a change in stiffness of up to $70 \%$ depending on the boundary conditions, load- and measuring directions. The height of the CARLOS TC load has significantly increased compared to usual testing conditions in order to evoke an early failure of selected spot welds. The already introduced DegraRP approach for uniaxial loaded specimens has been extended considering the altering stress state at each spot weld and the effects of multiaxiality on the degradation behavior. The extended approach has proven feasible and quite accurate in the prediction of local strain redistributions due to stiffness changes of single spot welds.

However there still are certain limitations to the methodology as changes in the stiffness of the base material (induced by cracks) or geometrical nonlinearities cannot be considered at the moment. Additionally the underlying material parameter set needs further improvement and refinement. In terms of automatization and performance the implementation still leaves room for improvement. At the moment the computation is conducted using a fixed step size (e.g. $n / n_{\text {ref }}=25 \%$ ). In future extensions the computation parameters (used for uniaxial loading) need to be adapted to multiaxial, variable amplitude loading.

\section{References}

[1] J. Meschke, J. Tölle, L. Berger, ATZ, Automobiltech. Z., 11, 48-53 (2017)

[2] P. Rösch, T. Bruder, T. Bein, Procedia Eng., 213, 437-447 (2018)

[3] A. H. Fritz, G. Schulze, Fertigungstechnik (Berlin, 2008)

[4] T. Seeger, S. Greuling, J. Brüning, P. Leis, C. M. Sonsino, D. Radaj, FAT, 196 (2015)

[5] J. Draisma, J. L. Overbeeke, Met. Constr. Br. Weld. J., 7, 213-219 (1974)

[6] R. Yuuki, T. Ohira, H. Nakatsukasa, W. Yi, JSMET, 467, 1772-1779 (1985)

[7] T. Satoh, H. Abe, K. Nishikikawa, M. Morita, Trans. Jpn. Weld. Soc., 1, 46-51 (1991)

[8] A. Rupp, K. Störzel, V. Grubisic, SAE Trans, 104, 635-645 (1995)

[9] H. Hanselka, K. Störzel, T. Bruder, FAT, 239 (2012)

[10] Y. Nakahra, M. Takahashi, A. Kawamoto, M. Fujimotot, N. Tomioka, SAE 2000 World Congress (2000)

[11] P. Rösch, T. Bruder, P. Wagner, Materialwiss. Werkstofftech. (to be published)

[12] M. Hack, S. Straesser, LMS Virtual.Lab Durability Solver Training (Kaiserslautern, 2016)

[13] T. Bruder, H. Klätsche, A. Sigwart, J. Riehle, Materialprüfung, 47, 429-435 (2005) 